\title{
CTENOPHTHALMUS (CTENOPHTHALMUS) STIRPS n. sp. DE TURQUIE (SIPHONAPTERA, HYSTRICHOPSYLLIDAE)
}

\author{
J. C. BEAUCOURNU* et V. ORHAN**
}

RÉSUMÉ. Description de Ctenophthalmus (Ctenophthalmus) stirps n. sp. ; cette puce appartient au sous-groupe andorrensis (Smit, 1963).

\section{Ctenophthalmus (Ctenophthalmus) stirps n. sp. from Turkey} (Siphonaptera, Hystrichopsyllidae).

SUMMARY. Ctenophthalmus (Ctenophthalmus) stirps n. sp. (andorrensis sub-group, Smit 1963) is described.

Une précédente enquête (Orhan et Beaucournu, 1982) avait signalé sept Siphonaptères inféodés aux micromammifères en Anatolie occidentale. La poursuite de ce travail dans la province de Kütahya (300 km à l'O.-S.O. d'Ankara) vient de mettre en évidence une espèce nouvelle que nous décrivons ci-après.

\section{Matériel}

Mâle holotype, femelle allotype, sur Pitymys majori (Thomas 1906), Ogrulea, Murat Dagi (Vilayet Kütahya) (Turquie), $1600 \mathrm{~m}$, août 1981 (V. O. rec.).

Holotype et Allotype sont dans les collections du premier signataire (Collections ultérieurement déposées au Muséum National d'Histoire Naturelle, Laboratoire d'Entomologie).

Derivatio nominis : du latin stirps, souche d'une lignée, mot mis en apposition.

* Laboratoire de Parasitologie (Entomologie médicale), U.E.R. médicales et pharmaceutiques, avenue du Prof. Léon-Bernard, F 35000 Rennes.

** Laboratoire de Parasitologie, Faculté de Médecine, Bornova-Izmir (Turquie).

Accepté le 27 octobre 1982 . 


\section{Description}

Ctenophthalmus s. st., appartenant au sous-groupe andorrensis (Smit 1963). Segments génitaux seuls caractéristiques.

Mâle: Processus basimeris dorsalis (fig. 1) triangulaire portant 2 longues soies

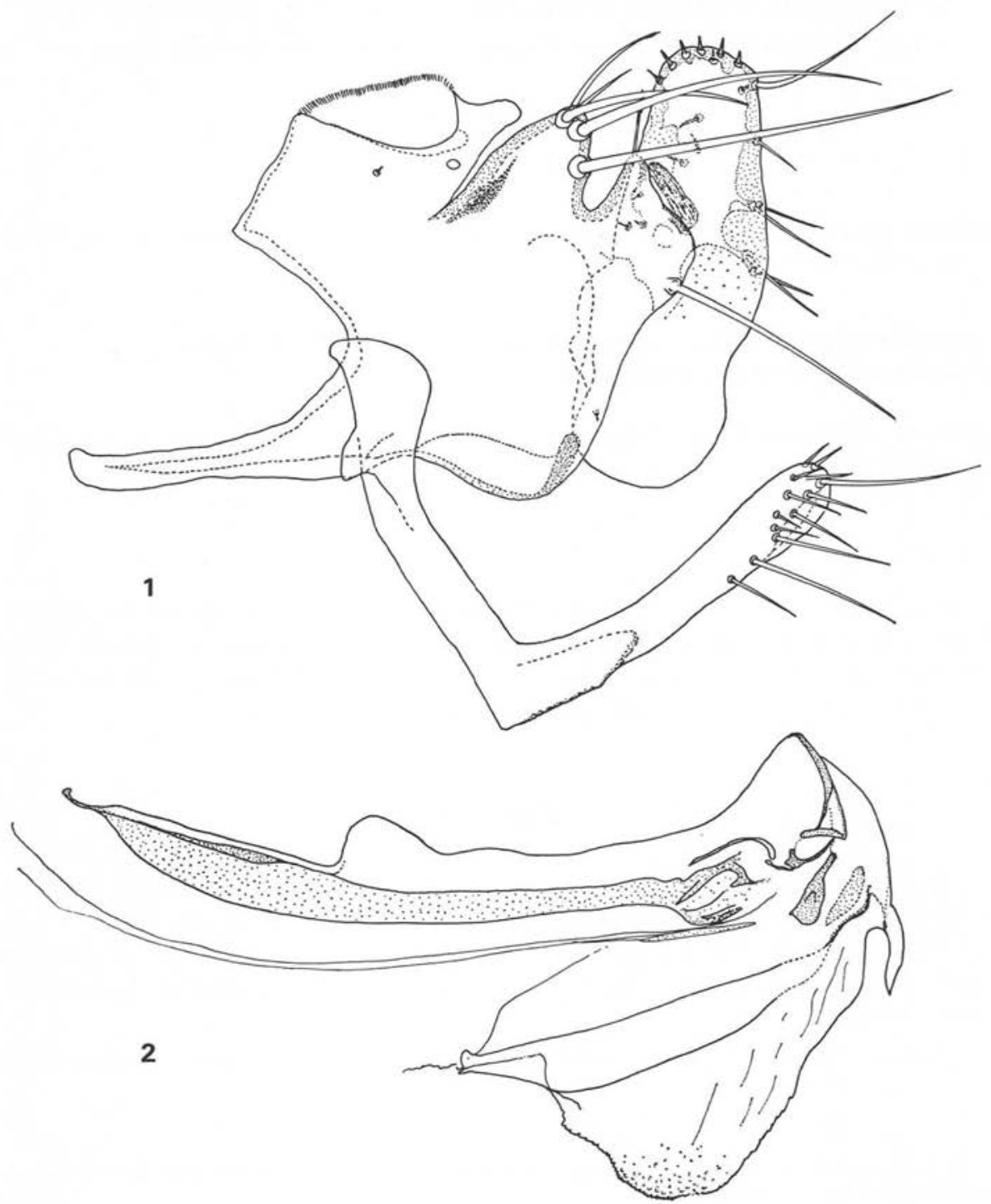

FIG. I. - Ctenophthalmus (Ctenophthalmus) stirps n. sp., Holotype, segment IX ;

FIG. 2 : $d^{\circ}$, phallosome. 
marginales. Processus basimeris ventralis nettement plus court que le p.b.d., montrant une ébauche de lobulation. Soie acétabulaire longue. Processus telomeris allongé, à peine moins large à l'apex qu'à la base. Apex large et arrondi entièrement occupé par l'insertion des 5 sensilles. Soie la plus basse de la marge postérieure insérée audessus du milieu. Sternite IX : bras distal allongé à bords subparallèles, apex arrondi, zone hyaline très étroite. Phallosome ( $f i g$. 2) à grande lamella, pendante, finement écailleuse (1).

Femelle: Très proche, non seulement des autres espèces du sous-groupe andorrensis, mais aussi de $C$. fransmiti Suciu, espèce avec laquelle elle cohabite ; il est vraisemblable que certains exemplaires isolés ne sont pas séparables.

Sternite VII (fig. 3) caractérisé par un lobe dorso-médian arrondi surplombant une concavité irrégulière et un petit lobe ventral ; il ne présente pas, au niveau de la concavité, le petit lobe triangulaire présent chez les allotypes de $C$. a. andorrensis Smit 1961, C. a. catalaniensis Beaucournu 1973 et C. bifidatus Smit 1960. Toutefois

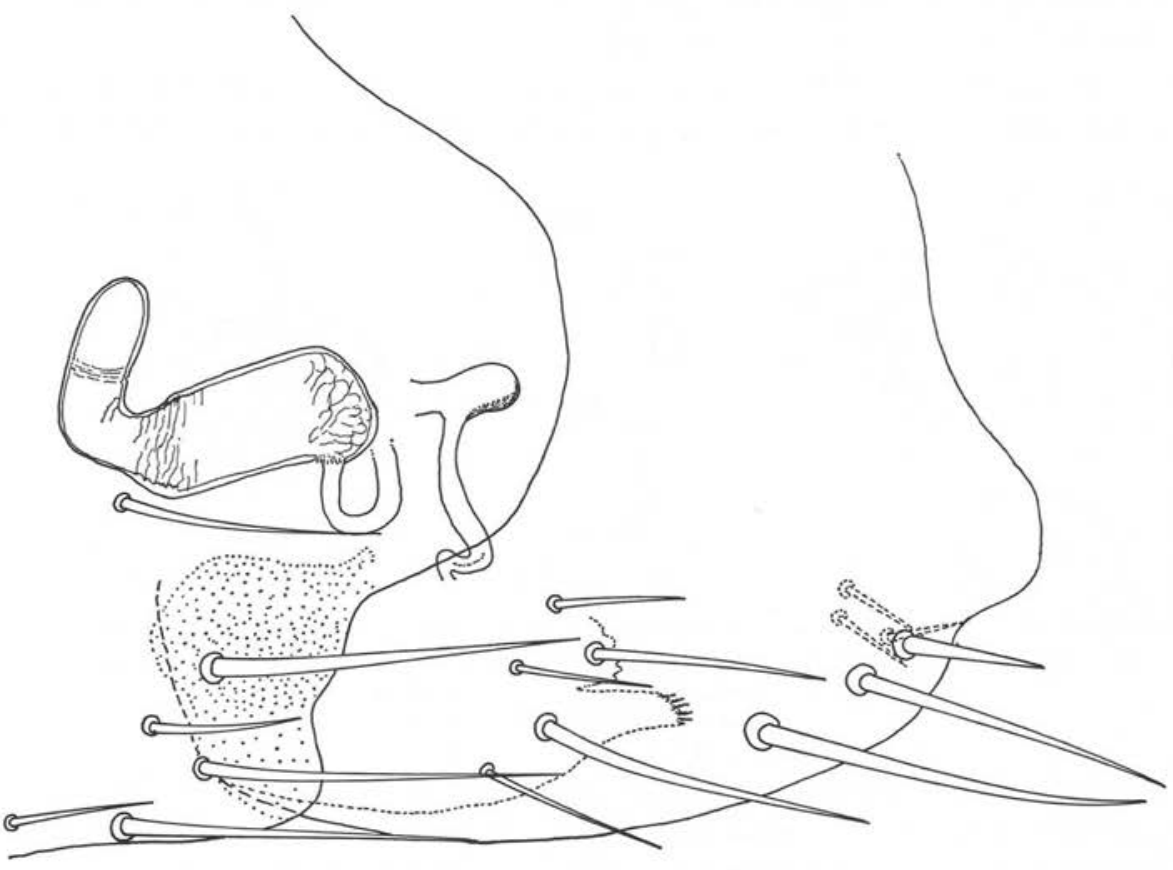

3

FIG. 3. - Ctenophthalmus (Ctenophthalmus) stirps n. sp., Allotype, sternites VII et VIII, tergite VIII, spermathèque.

I. Elle ne montre pas la structure observée par Peus (I976) chez C. bifidatus mais non mention rée dans la description originale. 
Beaucournu (1973) a montré que certains taxa de ce sous-groupe en étaient dépourvus dans plus de $90 \%$ des cas : ce critère ne pourra donc acquérir une valeur que sur une grande série de spécimens. Tergite VIII : proche de celui de $C$. bifidatus avec une légère concavité pré-apicale. Spermathèque et conduits génitaux non distinctifs.

\section{Discussion}

L'appartenance de $C$. stirps n. sp. au sous-groupe andorrensis est évidente et particulièrement affirmée par la structure du phallosome. Deux espèces y étaient incluses : $C$. andorrensis et $C$. bifidatus. La première est un Ctenophthalmus s. st. typique, évoquant par son segment IX (mâle) le sous-groupe agyrtes; la seconde se singularise par son télomère, non élargi à la base et surtout par son sternite IX dont la branche distale est bifurquée (1). Chez $C$. stirps n. sp., le basimère est si proche de celui de $C$. andorrensis que si nous l'avions récolté dans l'aire de cette dernière nous aurions été tentés de n'en faire qu'une sous-espèce. Toutefois le télomère étroit à la base et à apex large, la rapproche de $C$. bifidatus.

C. andorrensis, polytypique, a une répartition essentiellement ibérique ( $f i g .4)$ : il est connu de la Catalogne française au Maroc central, à travers toute la partie

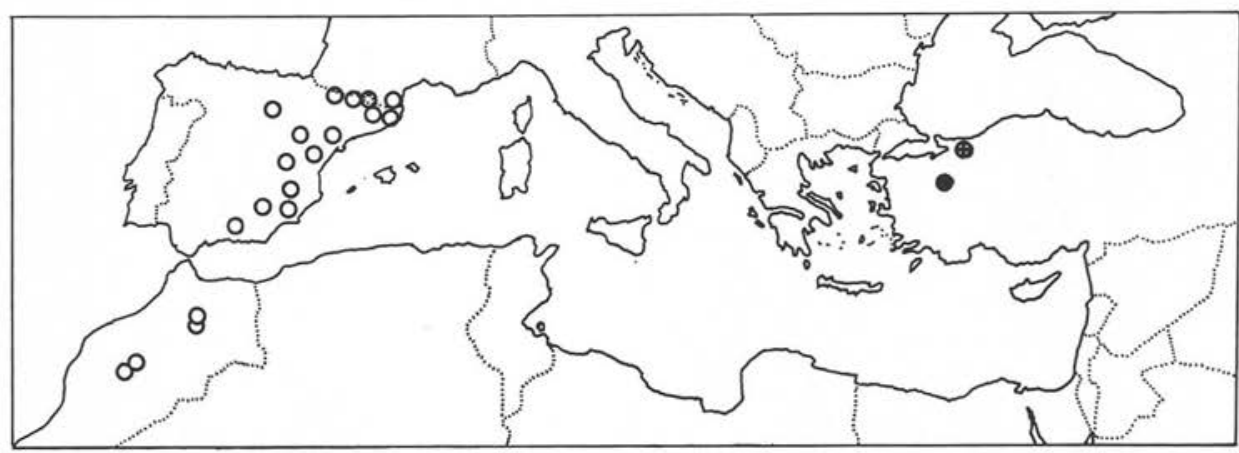

FIG. 4. - Carte du bassin méditerranéen : répartition connue des Ctenophthalmus du sous-genre andorrensis : o C. andorrensis ssp.,

$\oplus$ C. bifurcatus, $\bullet$. stirps.

orientale de la Péninsule ibérique. $C$. bifidatus n'est signalé que de Turquie et les quelques prélèvements opérés jusqu'à présent tendent à faire penser qu'il s'agit, comme bon nombre d'autres Ctenophthalmus, d'une espèce à endémisme étroit, limitée à la province de Bolü (Peus, 1976). Cette région est relativement proche de celle où nous avons récolté $C$. stirps $\mathrm{n}$. $\mathrm{sp}$. (230 km à vol d'oiseau). Il est hautement vraisemblable que notre nouvelle espèce est directement affiliée à bifidatus qui s'en serait séparé par une mutation.

I. Ce qui est un caractère extrêmement aberrant. 
Beaucournu (1976) a évoqué brièvement la répartition du sous-groupe andorrensis scindé en deux plages occupant chacune une extrémité du Bassin méditerranéen. L'origine du genre étant vraisemblablement la zone caucasienne (Beaucournu, op. cit.), C. stirps n. sp. apparaît, actuellement, comme la forme souche de ce sousgroupe ; $C$. bifidatus s'en détache sur place, et sans doute récemment; $C$. andorrensis est un migrant préglaciaire, isolé ultérieurement dans la Péninsule ibérique où il a évolué donnant 3 formes très proches, qui ne sont peut-être en fait qu'une évolution clinale, du Nord au Sud de cette région (Beaucournu et coll., 1973). Il est vraisemblable que la présence de cette espèce au Maroc (Hastriter et Tipton, 1975) où elle est représentée par le même taxon que dans le sud de l'Espagne, n'est qu'un phénomène récent, historique (Beaucournu, op. cit).

\section{BIBLIOGRAPHIE}

Beaucournu J. C. : Quatre siphonaptères nouveaux pour la faune française. Description de Ctenophthalmus (C.) andorrensis catalaniensis ssp. nova. Bull. Soc. Scient. Bretagne, 1972 (1973), 47, I69-175.

Beaucournu J. C. : Contribution à l'étude des Siphonaptères de Mammifères du nord-ouest de la région méditerranéenne (France, Italie, péninsule Ibérique). Thèse Doct. ès sciences, Rennes, I976, 284 p., cartes et tableaux.

Beaucournu J. C., Gilot B., Vericad J. R. : Deux Ctenophthalmus nouveaux (Siphonaptera, Hystrichopsyllidae) du Sud-Est ibérique. Rev. Iber. Parasitol., I973, 33, I27-134.

Hastriter M. W., Tipton V. J. : Fleas (Siphonaptera) associated with small mammals of Morocco. Jl. Egypt. Health Assoc., 1975, so, 79-169.

Orhan V., Beaucournu J. C. : Sur quelques Siphonaptères de Turquie occidentale. Bull. Soc. Entom. France, 1982, 87, 312-318.

Peus F. : Flöhe aus Anatolien und anderen Ländern des Nahen Ostens. Abhandl. Zool.-Bot. Gesellschaft Wien, 1976 (1977), 20, 1 iा $\mathrm{p}$.

Smrt F. G. A. M. : Species-groups in Ctenophthalmus (Siphonaptera : Hystrichopsyllidae). Bull. Brit. Mus. (Nat. Hist.) Entom., 1963, 14, 107-152. 